\title{
Acute allergic reaction after intravenous saline injection: an unusual presentation of chlorhexidine allergy
}

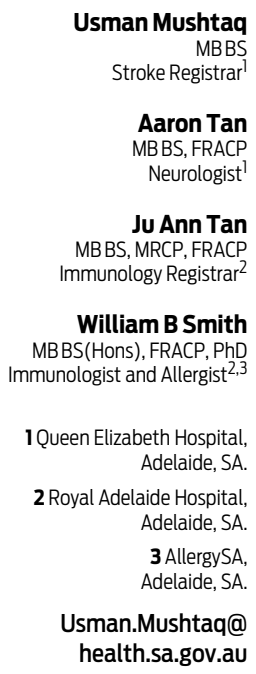

doi: 10.5694/mjal3.00144

\section{Clinical record}

A 63-year-old man presented to the emergency department after an episode of transient left hemiparesis and hemianaesthesia. His only history of allergy was an episode of mild urticaria after a postsurgical fentanyl infusion a few years previously. An intravenous cannula was inserted in his left cubital fossa. His neurological symptoms had resolved completely by the time he was reviewed by the neurology registrar. A magnetic resonance imaging brain scan was planned. The intravenous cannula was flushed with $10 \mathrm{~mL}$ of $0.9 \%$ normal saline after the rubber cannula connector was wiped with an alcohol-based swab. Within minutes, the patient experienced rapid development of generalised urticaria and periorbital oedema. He was treated with $25 \mathrm{mg}$ of promethazine and $100 \mathrm{mg}$ of intravenous hydrocortisone, with a good response. He had been fasting for 4 hours before this and was given no other medication.

Three days later, the patient was challenged with the same brand and batch of normal saline through a new intravenous cannula inserted in a different site. The cannula connector was again wiped with the same brand of alcohol-based swab before the challenge. A similar reaction, with immediate generalised urticaria (Figure), was produced, raising the suspicion of allergy to normal saline. Four hours after the challenge, his serum tryptase level was normal.

The patient was referred for allergy testing. Latex allergy was ruled out through negative skin prick testing and serum-specific IgE. He also tested negative on skin prick and intradermal testing to the specific brand of normal saline that had been used, and there were no additives found in the normal saline. He was then challenged with normal saline given through the same brand of intravenous cannula, with a negative result, thus ruling out hypersensitivity to a coating on the cannula. Finally, he underwent a skin prick test to chlorhexidine $0.1 \%$, which yielded a strongly positive reaction ( $16 \mathrm{~mm}$ wheal, $35 \mathrm{~mm}$ flare). It was found that the alcohol-based swab used to wipe the cannula connector contained $70 \%$ isopropyl alcohol and $2 \%$ chlorhexidine. Chlorhexidine allergy was confirmed with a positive chlorhexidine-specific lgE test (1.0 kU/L; class 2).

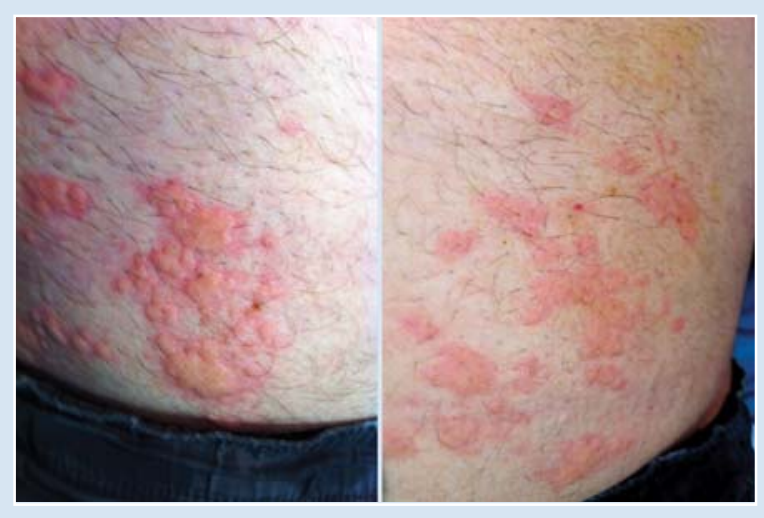

Allergic reaction immediately after normal saline challenge. hlorhexidine is widely used as an antiseptic solution in health care settings and in products such as mouthwash, disinfectants and toothpastes. An increase in its use in health care settings in recent years has led to increasing reports of chlorhexidine hypersensitivity reactions. Such reactions may be immediate or delayed, ${ }^{1,2}$ and acute reactions reflect type 1 hypersensitivity mediated by chlorhexidine-specific IgE. The incidence of immediate hypersensitivity to chlorhexidine is still unknown, ${ }^{1}$ but it has been increasingly reported in relation to exposures including mouthwash, ${ }^{3}$ anaesthetic lubricants ${ }^{4,5}$ and chlorhexidine-coated venous catheters. ${ }^{6}$ As reactions can include contact dermatitis, urticaria and life-threatening anaphylaxis, ${ }^{7}$ it is paramount that chlorhexidine allergy be identified. Chlorhexidine allergy should be considered in patients who experience an allergic reaction to an intravenous injection.

In this case, the cause was not immediately evident, as the chlorhexidine was contained in the alcohol-based swab used to wipe the intravenous administration port. The patient's reaction was initially thought to be caused by hypersensitivity to normal saline, which has been reported only twice in the literature. ${ }^{8,9}$ Normal saline is isotonic sodium chloride and should not cause an allergic reaction unless there is an additive in the solution. The published reports of normal saline allergy did not include skin prick or intradermal tests, and other potential causes were not adequately excluded. It was only after a series of investigations that our patient was found to be allergic to chlorhexidine. We postulate that a small amount of chlorhexidine from the swab was carried from the surface of the connector through the intravenous cannula while flushing it with normal saline. The patient was likely sensitised to chlorhexidine from exposure during previous hospital admissions. He was issued with a MedicAlert bracelet to prevent future exposure to chlorhexidine in the health care setting and was advised to avoid using mouthwashes and other products containing chlorhexidine.

The amount of chlorhexidine exposure was very small (only $2 \%$ in the swab) in this case, which resulted in a significant but not dangerous reaction. Greater exposure to chlorhexidine, such as in surgical preparation or insertion of a coated cannula, could have led to life-threatening anaphylaxis. Skin prick testing with chlorhexidine was positive in this patient but has relatively low sensitivity; intradermal testing with diluted chlorhexidine is more sensitive. Blood 


\section{Lessons from practice}

- As chlorhexidine is becoming more widely used in health care settings because of its superior antiseptic properties, serious chlorhexidine allergic reactions are being increasingly described.

- Chlorhexidine allergy should be considered in the differential diagnosis of an allergic reaction occurring after administration of any fluid or drug by intravenous injection.

- Acute allergic reactions to chlorhexidine are lgE-mediated, and diagnosis can be confirmed through skin prick testing by an allergy specialist or by a blood test for chlorhexidine-specific IgE.

- Allergic patients need to be exempted from chlorhexidine exposure in health care settings, with use of substitutes such as alcohol or povidone-iodine.

testing for chlorhexidine-specific IgE has high diagnostic sensitivity and specificity. ${ }^{1}$

Recent evidence-based guidelines have emphasised the superior antibacterial properties of chlorhexidine compared with alcohol and povidone-iodine. ${ }^{10}$ This has led to recommendations for use of chlorhexidine-based products in hospital protocols as a matter of policy. While allergy to chlorhexidine is rare, incidents are likely to increase if the use of chlorhexidine in health care settings becomes more widespread, and the potential for mild or severe acute allergic reactions (anaphylaxis) after chlorhexidine exposure must be borne in mind. Provisions will need to be made to exempt allergic patients from exposure to chlorhexidine, with use of substitutes such as alcohol or povidone-iodine.
Competing interests: No relevant disclosures.

1 Calogiuri GF, Di Leo E, Trautmann A, et al. Chlorhexidine hypersensitivity: a critical and updated review. J Allergy Ther 2013; 4: 141. doi: 10.4172/21556121.1000141.

2 Beaudouin E, Kanny G, Morisset M, et al. Immediate hypersensitivity to chlorhexidine: literature review. Eur Ann Allergy Clin Immunol 2004; 36: 123-126.

3 Sharma A, Chopra H. Chlorhexidine urticaria: a rare occurrence with a common mouthwash. Indian J Dent Res 2009; 20: 377-379.

4 Parkes AW, Harper N, Herwadkar A, Pumphrey R. Anaphylaxis to chlorhexidine component of Instillagel: a case series. Br J Anaesth 2009; 102: 65-68.

5 Bae YJ, Park CS, Lee JK, et al. A case of anaphylaxis to chlorhexidine during digital rectal examination. J Korean Med Sci 2008; 23: 526-528.

6 Khoo A, Oziemski P. Chlorhexidine impregnated central venous catheter inducing anaphylactic shock in the intensive care unit. Heart Lung Circ 2011; 20: 669-670.

7 Khan RA, Kazi T, O'Donohoe B. Near fatal intra-operative anaphylaxis to chlorhexidine - is it time to change practice? BMJ Case Rep 2011; 2011: bcr0920092300.

8 Ay D, Aktas C, Sarikaya S, Cetin A. An unusual cause of allergy: case report of normal saline solution allergy. Am J Emerg Med 2009; 27: 130.el-130.e2.

9 Litvin ME, Shemchuck AS, Lisetski VA. [Anaphylactic shock caused by intravenous injection of isotonic solution of sodium chloride] [Russian]. Klin Khir 1976; (7): 59-61.

10 O'Grady NP, Alexander M, Burns LA, et al; Healthcare Infection Control Practices Advisory Committee. Guidelines for the prevention of intravascular catheter-related infections, 2011. Atlanta: Centers for Disease Control and Prevention, 2011. http://www.cdc.gov/hicpac/pdf/guidelines/ bsi-guidelines-2011.pdf (accessed Mar 2014). 